Groups Geom. Dyn. 4 (2010), 739-757

DOI $10.4171 / \mathrm{GGD} / 103$
Groups, Geometry, and Dynamics

(C) European Mathematical Society

\title{
Automorphisms of partially commutative groups I: Linear subgroups*
}

\author{
Andrew J. Duncan, Ilya V. Kazachkov and Vladimir N. Remeslennikov
}

\begin{abstract}
We construct and describe several arithmetic subgroups of the automorphism group of a partially commutative group. More precisely, given an arbitrary finite graph $\Gamma$ we construct arithmetic subgroups $\operatorname{St}\left(L_{Y}\right)$ and $\operatorname{St}\left(L^{\max }\right)$, represented as subgroups of $\operatorname{GL}(n, \mathbb{Z})$, where $n$ is the number of vertices of the graph $\Gamma$. Here $L_{Y}$ and $L^{\max }$ are certain lattices of subsets of $X=V(\Gamma)$ and $\operatorname{St}(K)$ is the stabiliser of the subgroup generated by $K$. In addition we give a description of the decomposition of the group $\mathrm{St}^{\mathrm{conj}}\left(L_{X}\right)$, which stabilises $L_{X}$ up to conjugacy, as a semidirect product of the group of conjugating automorphisms and $\operatorname{St}\left(L_{X}\right)$.
\end{abstract}

Mathematics Subject Classification (2010). 20F38, 20E36, 20F36, 20 G05.

Keywords. Partially commutative groups, right-angled Artin groups, automorphism groups, arithmetic groups.

\section{Introduction}

Recently a wave of interest in groups of automorphisms of partially commutative groups has risen; see [3], [2], [4], [10]. This emergence of interest may be attributable to the fact that numerous geometric and arithmetic groups are subgroups of these groups.

The goal of this paper is to construct and describe certain arithmetic subgroups of the automorphism group of a partially commutative group. More precisely, given an arbitrary finite graph $\Gamma$ we construct an arithmetic subgroup $\operatorname{St}(L(\Gamma))$ (see Section 2.1 for definitions), represented as a subgroup of $\operatorname{GL}(n, \mathbb{Z})$, where $n$ is the number of vertices of the graph $\Gamma$; see Theorems 2.4 and 2.12. Note that our proof is independent of the results of Laurence and Serviatius, [11], [12], which give a description of the generating set of the automorphism group $\operatorname{Aut}(G(\Gamma))$. One of the advantages of this proof is that it is largely combinatorial, rather than group-theoretic, so could be adjusted to obtain analogous results for partially commutative algebras determined by the graph $\Gamma$ (in various varieties of algebras).

\footnotetext{
* Research carried out while the second and third named authors were visiting Newcastle University with the support of EPSRC grant EP/D065275/1, June 2005.
} 
In the last section of the paper we give a description of the decomposition of the group $\operatorname{St}^{\text {conj }}(L(\Gamma))$ (see Section 2.1 for definitions) as a semidirect product of the group of conjugating automorphisms $\operatorname{Conj}(G)$ and $\operatorname{St}(L(\Gamma))$. This result is closely related to Theorem 1.4 of [10], but the situations considered in [10] and in this paper are somewhat different.

The authors are grateful to the Centre de Recerca Matemàtica (CRM): these results were announced at a workshop, supported by the CRM, in Manresa, and the proof outlined in seminars, while the authors were on research visits to the CRM in September 2006.

\section{Preliminaries}

1.1. Graphs and lattices of closed subsets. In this section we give definitions and a summary of the facts we need concerning graphs, orthogonal systems and closed subsets of a graph. For further details the reader is referred to [7]. Graph will mean undirected, finite, simple graph throughout this paper. If $x$ and $y$ are vertices of a graph then we define the distance $d(x, y)$ from $x$ to $y$ to be the minimum of the lengths of all paths from $x$ to $y$ in $\Gamma$. A subgraph $S$ of a graph $\Gamma$ is called a full subgraph if vertices $a$ and $b$ of $S$ are joined by an edge of $S$ whenever they are joined by an edge of $\Gamma$.

Let $\Gamma$ be a graph with $V(\Gamma)=X$. A subset $Y$ of $X$ is called a simplex if the full subgraph of $\Gamma$ with vertices $Y$ is isomorphic to a complete graph. Given a subset $Y$ of $X$ the orthogonal complement of $Y$ is defined to be

$$
Y^{\perp}=\{u \in X \mid d(u, y) \leq 1 \text { for all } y \in Y\} .
$$

By convention we set $\emptyset^{\perp}=X$. It is not hard to see that $Y \subseteq Y^{\perp \perp}$ and $Y^{\perp}=$ $Y^{\perp \perp \perp}$ [7], Lemma 2.1. We define the closure of $Y$ to be $\operatorname{cl}(Y)=Y^{\perp \perp}$. The closure operator in $\Gamma$ satisfies, among others, the properties that $Y \subseteq \operatorname{cl}(Y), \operatorname{cl}\left(Y^{\perp}\right)=Y^{\perp}$ and $\operatorname{cl}(\operatorname{cl}(Y))=\operatorname{cl}(Y)$ [7], Lemma 2.4. Moreover if $Y_{1} \subseteq Y_{2} \subseteq X$ then $\operatorname{cl}\left(Y_{1}\right) \subseteq$ $\operatorname{cl}\left(Y_{2}\right)$.

Definition 1.1. A subset $Y$ of $X$ is called closed (with respect to $\Gamma$ ) if $Y=\operatorname{cl}(Y)$. Denote by $L=L(\Gamma)$ the set of all closed subsets of $X$.

Then $\operatorname{cl}(Y) \in L$, for all $Y \subseteq X$ and $U \in L$ if and only if $U=V^{\perp}$ for some $V \subseteq X$ [7], Lemma 2.7. The relation $Y_{1} \subseteq Y_{2}$ defines a partial order on the set $L$. As the closure operator $\mathrm{cl}$ is inclusion preserving and maps arbitrary subsets of $X$ into closed sets, $L$ is a lattice where the infimum $Y_{1} \wedge Y_{2}$ of $Y_{1}$ and $Y_{2}$ is $Y_{1} \wedge Y_{2}=\operatorname{cl}\left(Y_{1} \cap Y_{2}\right)=Y_{1} \cap Y_{2}$ and the supremum is $Y_{1} \vee Y_{2}=\operatorname{cl}\left(Y_{1} \cup Y_{2}\right)$.

1.2. Partially commutative groups. Let $\Gamma$ be a finite, undirected, simple graph. Let $X=V(\Gamma)$ be the set of vertices of $\Gamma$ and let $F(X)$ be the free group on $X$. For 
elements $g, h$ of a group we denote the commutator $g^{-1} h^{-1} g h$ of $g$ and $h$ by $[g, h]$. Let

$$
R=\left\{\left[x_{i}, x_{j}\right] \in F(X) \mid x_{i}, x_{j} \in X \text { and there is an edge from } x_{i} \text { to } x_{j} \text { in } \Gamma\right\} .
$$

We define the partially commutative group with (commutation) graph $\Gamma$ to be the group $G(\Gamma)$ with presentation $\langle X \mid R\rangle$. (Note that these are the groups which are called finitely generated free partially commutative groups in [5].)

The subgroup generated by a subset $Y \subseteq X$ is called a canonical parabolic subgroup of $G$ and denoted $G(Y)$. This subgroup is equal to the partially commutative group with commutation graph the full subgraph of $\Gamma$ generated by $Y$ [1]. The connection between closed sets and the group $G(\Gamma)$ is established by Proposition 3.9 of [8]: a subgroup $G(Y)^{g}$ of $G$ is a centraliser if and only if $Y \in L(\Gamma)$. If $Y$ is a closed subset of $\Gamma$ and $g \in G$ then the subgroup $G(Y)^{g}=C_{G}\left(Y^{\perp}\right)^{g}$ is called a parabolic centraliser.

Let $\Gamma$ be a simple graph, $G=G(\Gamma)$ and let $w \in G$. Denote by $\lg (w)$ the minimum of the lengths of words that represents the element $w$. We say that $w \in G$ is cyclically minimal if and only if

$$
\lg \left(g^{-1} w g\right) \geq \lg (w) \quad \text { for all } g \in G .
$$

We write $u \circ v$ to express the fact that $\lg (u v)=l(u)+l(v)$. We say that $u$ is a left divisor (right divisor) of $w$ if there exists $v$ such that $w=u \circ v(w=v \circ u)$. If $g \in G$ and $w$ is a word of minimal length representing $g$ then we write $\alpha(g)$ for the set of elements $x \in X$ such that $x^{ \pm 1}$ occurs in $w$. It is shown in [1] that $\alpha(g)$ depends only on $g$ and not on the choice of $w$.

The non-commutation graph of the partially commutative group $G(\Gamma)$ is the graph $\Delta$, dual to $\Gamma$, with vertex set $V(\Delta)=X$ and an edge connecting $x_{i}$ and $x_{j}$ if and only if $\left[x_{i}, x_{j}\right] \neq 1$. The graph $\Delta$ is a union of its connected components $\Delta_{1}, \ldots, \Delta_{k}$ and words that depend on letters from distinct components commute. For any graph $\Gamma$, if $S$ is a subset of $V(\Gamma)$ we shall write $\Gamma(S)$ for the full subgraph of $\Gamma$ with vertices $S$. Now, if the vertex set of $\Delta_{k}$ is $I_{k}$ and $\Gamma_{k}=\Gamma\left(I_{k}\right)$ then $G=G\left(\Gamma_{1}\right) \times \cdots \times G\left(\Gamma_{k}\right)$. For $g \in G$ let $\alpha(g)$ be the set of elements $x$ of $X$ such that $x^{ \pm 1}$ occurs in a minimal word $w$ representing $g$. Now suppose that the full subgraph $\Delta(\alpha(w))$ of $\Delta$ with vertices $\alpha(w)$ has connected components $\Delta_{1}, \ldots, \Delta_{l}$ and let the vertex set of $\Delta_{j}$ be $I_{j}$. Then, since $\left[I_{j}, I_{k}\right]=1$, we can split $w$ into the product of commuting words, $w=w_{1} \circ \cdots \circ w_{l}$, where $w_{j} \in G\left(\Gamma\left(I_{j}\right)\right)$, so $\left[w_{j}, w_{k}\right]=1$ for all $j, k$. If $w$ is cyclically minimal then we call this expression for $w$ a block decomposition of $w$ and say $w_{j}$ is a block of $w$ for $j=1, \ldots, l$. Thus $w$ itself is a block if and only if $\Delta(\alpha(w))$ is connected.

As in [8] we make the following definition. An element $g \in G$ is called a root element if it has the property that $g=h^{n}$, for some $h \in G$, implies that $n= \pm 1$.

Definition 1.2. Let $w$ be a cyclically minimal root element of $G$ with block decomposition $w=w_{1} \ldots w_{k}$ and let $Z$ be a subset of $X$ such that $Z \subseteq \alpha(w)^{\perp}$. Then 
the subgroup $Q=Q(w, Z)=\left\langle w_{1}\right\rangle \times \cdots \times\left\langle w_{k}\right\rangle \times G(Z)$ is called a canonical quasiparabolic subgroup of $G$.

A subgroup is called quasiparabolic if it is conjugate to a canonical quasiparabolic subgroup. In [8] centralisers of arbitrary subsets of a partially commutative group are characterised in terms of quasiparabolic subgroups and we shall use this result in Section 2.3.

1.3. Conjugating automorphisms. Automorphisms which act locally by conjugation play an important role in the structure of $\operatorname{Aut}(G)$.

For $S \subseteq X$ define $\Gamma_{S}$ to be $\Gamma \backslash S$, the graph obtained from $\Gamma$ by removing all vertices of $S$ and all their incident edges.

Definition 1.3. Let $x \in X$ and let $C$ be a connected component of $\Gamma_{x^{\perp}}$. The automorphism $\alpha_{C}(x)$ given by

$$
y \mapsto \begin{cases}y^{x} & \text { if } y \in C, \\ y & \text { otherwise }\end{cases}
$$

is called an elementary conjugating automorphism of $\Gamma$. The subgroup of $\operatorname{Aut}(G)$ generated by all elementary conjugating automorphisms (over all connected components of $\Gamma_{x^{\perp}}$ and all $x \in X$ ) is called the group of conjugating automorphisms and is denoted $\operatorname{Conj}(G)$.

Theorem 1.4 (M. Laurence [11]). An element $\psi \in \operatorname{Aut}(G)$ is called a conjugating automorphism if, for all $x \in X$, there exists $g_{x} \in G$ such that $x^{\psi}=x^{g_{x}}$.

From Theorem 1.4 it follows that the group of inner automorphisms $\operatorname{Inn}(G)$ is a subgroup of $\operatorname{Conj}(G)$; and is therefore a normal subgroup.

\section{Stabilisers of parabolic centralisers}

2.1. Stabiliser subgroups. Throughout the remainder of this paper let $\Gamma$ be a finite graph with vertices $X$, let $G=G(\Gamma)$ and let $L=L(\Gamma)$ be the lattice of closed sets of $\Gamma$. We denote the automorphism group of $G$ by $\operatorname{Aut}(G)$.

Definition 2.1. The stabiliser of $L$ is defined to be

$$
\operatorname{St}(L)=\left\{\phi \in \operatorname{Aut}(G) \mid G(Y)^{\phi}=G(Y) \text { for all } Y \in L\right\}
$$

and the conjugate-stabiliser of $L$ is defined to be

$$
\begin{gathered}
\operatorname{St}^{\text {conj }}(L)=\left\{\phi \in \operatorname{Aut}(G) \mid \text { for all } Y \in L \text { there exists } g_{Y}\right. \text { such that } \\
\left.\qquad G(Y)^{\phi}=G(Y)^{g_{Y}}\right\} .
\end{gathered}
$$


If $\phi \in \mathrm{St}^{\text {conj }}(L), Y \in L$ and $g_{Y}$ is such that $G(Y)^{\phi}=G(Y)^{g_{Y}}$ then we say that $\phi$ acts as $g_{Y}$ on $Y$.

Proposition 2.2. Both the stabiliser $\operatorname{St}(L)$ and the conjugate-stabiliser $\mathrm{St}^{\mathrm{conj}}(L)$ of $L$ are subgroups of $\operatorname{Aut}(G)$ and $\operatorname{St}(L)<\mathrm{St}^{\text {conj }}(L)$.

Proof. It is clear that the stabiliser of $L$ is a group and that it is contained in the conjugate-stabiliser. If $\phi \in \mathrm{St}^{\text {conj }}(L)$ acts as $g_{Y}$ on $Y \in L$ then $G(Y)=\left(G(Y)^{h}\right)^{\phi}$, where $h=\left(g_{Y}^{-1}\right)^{\phi^{-1}}$. Thus $G(Y)^{\phi^{-1}}=G(Y)^{h}$. If $\phi^{\prime} \in \mathrm{St}^{\text {conj }}(L)$ acts as $g_{Y}^{\prime}$ on $Y$ then $G(Y)^{\phi^{\prime} \phi}=G(Y)^{k}$, where $k=g_{Y}\left(g_{Y}^{\prime}\right)^{\phi}$.

2.2. Generators for the stabiliser of $\boldsymbol{L}$. We introduce three sets of maps $J, V_{\perp}$ and $\operatorname{Tr}_{\perp}$ : which will turn out to be automorphisms and to generate $\operatorname{St}(L)$ (cf. [12], [11]). First we recall some notation from [7] and establish some background information.

As in [7] we define an equivalence relation $\sim_{\perp}$ on $X$ by $x \sim_{\perp} y$ if and only if $x^{\perp}=y^{\perp}$. Denote the equivalence class of $x$ under $\sim_{\perp}$ by $[x]_{\perp}$. Then $[x]_{\perp}$ is a simplex for all $x \in X$. The set $N_{2}$ consists of those $x \in X$ such that $\left|[x]_{\perp}\right| \geq 2$. Define $N_{1}=X \backslash N_{2}, X^{\prime}=X / \sim_{\perp}$ and $N_{2}^{\prime}=\left\{[x]_{\perp} \in X^{\prime} \mid x \in N_{2}\right\}$. If $x \in N_{1}$ then $[x]_{\perp}=\{x\}$, so $X$ is the disjoint union

$$
X=\bigsqcup_{[x]_{\perp} \in N_{2}^{\prime}}[x]_{\perp} \sqcup N_{1} .
$$

For $x \in X$ we write $G[x]_{\perp}$ for $G\left([x]_{\perp}\right)=\left\langle[x]_{\perp}\right\rangle$, so $G[x]_{\perp} \subseteq G$. For all $x, y, z \in X$ such that $y \in[x]_{\perp}$ we have $[y, z]=1$ if and only if $[u, z]=1$ for all $u \in[x]_{\perp}$. It follows that we may extend an automorphism $\phi$ of $G[x]_{\perp}$ to an automorphism $\phi^{\varepsilon_{x}}$ of $G$ by setting $g^{\phi^{\varepsilon_{x}}}=g^{\phi}$ for all $g \in G[x]_{\perp}$, and $y^{\phi^{\varepsilon_{x}}}=y$ for all $y \in X \backslash[x]_{\perp}$. The map $\varepsilon_{x}$ such that $\phi \mapsto \phi^{\varepsilon_{x}}$ is then an monomorphism from $\operatorname{Aut}\left(G[x]_{\perp}\right)$ into $\operatorname{Aut}(G)$. Moreover, if $[x]_{\perp} \neq[y]_{\perp}$ then $G[x]_{\perp} \cap G[y]_{\perp}=1$. Therefore $\operatorname{Aut}\left(G[x]_{\perp}\right)^{\varepsilon_{x}} \cap \operatorname{Aut}\left(G[y]_{\perp}\right)^{\varepsilon_{y}}=1$ and, for all $\phi \in \operatorname{Aut}\left(G[x]_{\perp}\right)$ and $\psi \in \operatorname{Aut}\left(G[y]_{\perp}\right)$, we have $\phi^{\varepsilon_{x}} \psi^{\varepsilon_{y}}=\psi^{\varepsilon_{y}} \phi^{\varepsilon_{x}}$. Hence $\operatorname{Aut}(G)$ contains a subgroup

$$
V=\prod_{[x]_{\perp} \in N_{2}^{\prime}} \operatorname{Aut}\left(G[x]_{\perp}\right)^{\varepsilon_{x}} \times \prod_{x \in N_{1}} \operatorname{Aut}\left(G[x]_{\perp}\right)^{\varepsilon_{x}} .
$$

(1) The set $J$ consists of the extension to $G$ of all maps $X \rightarrow G$ such that $x^{\phi}=x$ or $x^{-1}$ for all $x \in X$. Then $J \leq \operatorname{Aut}(G)$ and $|J|=2^{|X|}$.

(2) We define

$$
V_{\perp}=\prod_{[x]_{\perp} \in N_{2}^{\prime}} \operatorname{Aut}\left(G[x]_{\perp}\right)^{\varepsilon_{x}} \leq V .
$$

(3) For distinct $x, y \in X$ define a map $\operatorname{tr}_{x, y}: X \rightarrow G$ by $\operatorname{tr}_{x, y}(x)=x y$, and $\operatorname{tr}_{x, y}(z)=z$ for $z \neq x$. If $x^{\perp} \backslash\{x\} \subseteq y^{\perp}$ then $\operatorname{tr}_{x, y}$ extends to an automorphism 
of $G$ which we also call $\operatorname{tr}_{x, y}$. Define $\operatorname{Tr}$ to be the set consisting of the extension to $G$ of all the maps $\operatorname{tr}_{x, y}$, where $x^{\perp} \backslash\{x\} \subseteq y^{\perp}$. Then $\operatorname{Tr} \subseteq \operatorname{Aut}(G)$. We define

$$
\operatorname{Tr}_{\perp}=\left\{\operatorname{tr}_{x, y} \in \operatorname{Tr} \mid \operatorname{cl}(y)<\operatorname{cl}(x)\right\} \subseteq \operatorname{Tr} .
$$

Note that we have excluded those $\operatorname{tr}_{x, y}$ where $\operatorname{cl}(y)=\operatorname{cl}(x)$ and that $\operatorname{cl}(y)<$ $\operatorname{cl}(x)$ implies that $y \in \operatorname{cl}(x)$ so that $x^{\perp} \subset y^{\perp}$ and $[x, y]=1$. Therefore the map $\operatorname{tr}_{x, y} \in \operatorname{Tr}_{\perp}$ if and only if $x^{\perp} \subset y^{\perp}$ (the inclusion being strict).

We remark that the subgroup generated by $J$ and $V_{\perp}$ is $V$. Moreover Laurence [11], building on results of Servatius [12], showed that $\operatorname{Aut}(G)$ is generated by $J, \operatorname{Tr}$ and $\operatorname{Conj}(G)$, together with automorphisms which permute the vertices of $\Gamma$ (see for example [7]).

Proposition 2.3. The sets of maps $J, V$ and $V_{\perp}$ are subgroups of $\operatorname{St}(L)$ and the set $\mathrm{Tr}_{\perp}$ is contained in $\operatorname{St}(L)$.

Proof. As $J$ fixes every parabolic subgroup it is clear that $J \leq \operatorname{St}(L)$. To see that $V_{\perp}$ is a subgroup of $\operatorname{St}(L)$ consider $Y \in L$. If $x \in Y$ and $z \in[x]_{\perp}$ then $Y^{\perp} \subseteq x^{\perp}=z^{\perp}$ so $z \in Y$. Hence $x \in Y$ implies that $[x]_{\perp} \subseteq Y$. Now suppose that $\phi \in V_{\perp}$. If $y \in Y \cap N_{2}$ then $y^{\phi} \in G[y]_{\perp} \leq G(Y)$, by the above. If $y \in Y \backslash N_{2}$ then $y^{\phi}=y \in G(Y)$. Hence $G(Y)^{\phi} \subseteq G(Y)$ and since $V_{\perp}$ is a subgroup of $\operatorname{Aut}(G)$ it follows that $G(Y)^{\phi}=G(Y)$. Therefore $V_{\perp} \leq \operatorname{St}(L)$. Finally, let $\tau=\operatorname{tr}_{x, y} \in \operatorname{Tr}_{\perp}$ and let $Y \in L$. If $x \notin Y$ then $\tau$ fixes $Y$ pointwise, so we assume that $x \in Y$. In this case $\operatorname{cl}(x) \subseteq Y$ and $y \in \operatorname{cl}(x)$, from the remark following the definition of $\operatorname{Tr}_{\perp}$. Hence $x^{\tau}=x y \in G(Y)$ and $G(Y)^{\tau} \leq G(Y)$. As $x=\left(x y^{-1}\right)^{\tau}$ it follows that $G(Y)^{\tau}=G(Y)$. As $V$ is generated by $J$ and $V_{\perp}$ it is also a subgroup of $\operatorname{St}(L)$.

Before stating the next theorem we shall briefly explain what is meant by an arithmetic group. Two subgroups $A$ and $B$ of a group $G$ are said to be commensurable if $A \cap B$ is of finite index in both $A$ and $B$. A linear algebraic group is a group which is also an affine algebraic variety, such that multiplication and inversion are morphisms of affine algebraic varieties. A linear algebraic group is said to be $\mathbb{Q}$-defined if it is a subgroup of $\operatorname{GL}(n, \mathbb{C})$ which can be defined by polynomials over $\mathbb{Q}$ and is such that the group operations are morphisms defined over $\mathbb{Q}$. Let $G$ be a $\mathbb{Q}$-defined algebraic group. A subgroup $A \subseteq G \cap \operatorname{GL}(n, \mathbb{Q})$ is called an arithmetic subgroup of $G$ if it is commensurable with $G \cap \operatorname{GL}(n, \mathbb{Z})$. A group is called arithmetic if it is isomorphic to an arithmetic subgroup of a $\mathbb{Q}$-defined linear algebraic group.

Theorem 2.4. The stabiliser $\operatorname{St}(L)$ is an arithmetic group generated by the elements of $J, V_{\perp}$ and $\operatorname{Tr}_{\perp}$.

We defer the proof of this theorem, which is part of the more technical Theorem 2.12 below. 
2.3. Ordering $\boldsymbol{L}$ and the stabiliser of $\boldsymbol{L}$-maximal elements. We shall now define a partial order on elements of $X$ which reflects the lattice structure of $L$. We shall then describe a subgroup of the automorphism group of $G$ which stabilises subgroups generated by closures of the maximal elements in this order. First note that if $Y \in L$ then $Y=\bigcup_{y \in Y} \operatorname{cl}(y)$. Therefore if $\phi \in \operatorname{Aut}(G)$ and $G(\operatorname{cl}(y))^{\phi}=G(\operatorname{cl}(y))$ for all $y \in Y$ then $G(Y)^{\phi}=G(Y)$. This implies that if $G(\operatorname{cl}(x))^{\phi}=G(\operatorname{cl}(x))$ for all $x \in X$ then $\phi \in \operatorname{St}(L)$. Setting $L_{X}=\{Y \in L \mid Y=\operatorname{cl}(x)$ for some $x \in X\}$ and $\operatorname{St}\left(L_{X}\right)=\left\{\phi \in \operatorname{Aut}(G) \mid G(Y)^{\phi}=G(Y)\right.$ for all $\left.Y \in L_{X}\right\}$ we have

$$
\operatorname{St}(L)=\operatorname{St}\left(L_{X}\right)
$$

Definition 2.5. Let $<_{L}$ be the partial order on $X$ given by $x<_{L} \quad y$ if and only if $\operatorname{cl}(x) \subset \operatorname{cl}(y)$ and $\operatorname{cl}(x) \neq \operatorname{cl}(y)$. By $x={ }_{L} y$ we mean $\operatorname{cl}(x)=\operatorname{cl}(y)$. We say $x$ is $L$-minimal (L-maximal) if $y \leq_{L} x\left(x \leq_{L} y\right)$ implies $\operatorname{cl}(y)=\operatorname{cl}(x)$.

Note that $y<_{L} x$ if and only if $\operatorname{cl}(y) \subseteq \operatorname{cl}(x)$ and $x \notin \operatorname{cl}(y)$.

Write $L^{\max }=\left\{\operatorname{cl}(x) \in L_{X} \mid x\right.$ is $<_{L}$-maximal $\}$ and denote $\operatorname{St}\left(L^{\max }\right)=\{\phi \in$ $\operatorname{Aut}(G) \mid G(Y)^{\phi}=G(Y)$ for all $\left.Y \in L^{\max }\right\}$.

Proposition 2.6. $\mathrm{St}(L)$ and $\mathrm{St}\left(L^{\mathrm{max}}\right)$ are commensurable.

Proof. Since $\operatorname{St}(L) \subseteq \operatorname{St}\left(L^{\max }\right)$, it suffices to prove that $\operatorname{St}(L)$ has finite index in $\operatorname{St}\left(L^{\max }\right)$. Let $\phi \in \operatorname{St}\left(L^{\max }\right)$, let $x \in X$, let $Z \in L^{\max }$, say $Z=\operatorname{cl}(z)$ for some $L$-maximal element $z \in X$, and let $Y \in L$ such that $Y \subseteq Z$. By definition of $\operatorname{St}\left(L^{\max }\right)$ we have $G(Z)^{\phi}=G(Z)$ and so $G(Y)^{\phi} \subseteq G(Z)^{\phi}=G(Z)$. As $Y \in L$ we have $Y=U^{\perp}$ for some $U \in L$, so $G(Y)=C_{G}(U)$, a canonical parabolic centraliser. Hence $G(Y)^{\phi}$ is a centraliser and from [8], Theorem 3.12, is conjugate to a quasiparabolic subgroup. As $G(Z)$ is Abelian it follows (loc. cit.) that $G(Y)^{\phi}$ is a canonical parabolic centraliser: that is $G(Y)^{\phi}=G(V)$ for some $V \in L$ with $V \subseteq G(Z)$. As $\phi$ is an automorphism it therefore permutes the subgroups $G(Y)$ where $Y$ is an element of the set $L(Z)=\{V \in L \mid V \subseteq Z\}$ and this induces a permutation on the set $L(Z)$. This holds for all elements $Z \in L^{\max }$ and setting $M=\bigcup_{Z \in L^{\max }} L(Z)$ we obtain a permutation $\sigma(\phi)$ of $M$. It is clear that if $\psi$ is another element of $\operatorname{St}\left(L^{\max }\right)$ then $\sigma(\phi \psi)=\sigma(\phi) \sigma(\psi)$. Thus, writing $S_{M}$ for the permutation group of the finite set $M$, we may view $\sigma$ as a homomorphism $\sigma: \operatorname{St}\left(L^{\max }\right) \rightarrow S_{M}$. If $\phi \in \operatorname{ker}(\sigma)$ then $\sigma(\phi)$ fixes every element of $M$ and in particular every element of $L_{X}$. Hence $\operatorname{ker}(\sigma) \subseteq \operatorname{St}\left(L_{X}\right)$ and since from (2.1) we have $\operatorname{St}\left(L_{X}\right)=\operatorname{St}(L)$, this completes the proof.

2.4. Ordering $X$ and closures of elements. Using the order induced from $L$ we shall define a total order on $X$. This order will be used to index a basis of $\mathbb{Z}^{n}$ with respect to which elements of $\operatorname{St}(L)$ will be described as matrices. The ordering depends on the following stratification of the closures of single elements of $X$. 
Proposition 2.7. $[x]_{\perp}=\operatorname{cl}(x) \backslash\left\{u \in \operatorname{cl}(y) \mid y<_{L} x\right\}$ for all $x \in X$. In particular, if $x$ is L-minimal then $[x]_{\perp}=\operatorname{cl}(x)$.

Proof. First recall $\operatorname{cl}(z)=\operatorname{cl}(x)$ if and only if $z^{\perp}=x^{\perp}$ (as $Y^{\perp}=Y^{\perp \perp \perp}$ ), so $z \in[x]_{\perp}$ if and only if $\operatorname{cl}(z)=\operatorname{cl}(x)$. If $u \in \operatorname{cl}(y)$, where $y<_{L} x$ then $\operatorname{cl}(u) \leq$ $\operatorname{cl}(y)<\operatorname{cl}(x)$ so $u^{\perp} \neq x^{\perp}$. Hence $[x]_{\perp} \subseteq \operatorname{cl}(x) \backslash\left\{u \in \operatorname{cl}(y) \mid y<_{L} x\right\}$. On the other hand if $z \in \operatorname{cl}(x)$ then $\operatorname{cl}(z) \subseteq \operatorname{cl}(x)$. If also $z \notin \operatorname{cl}(y)$ for all $y<_{L} x$ then $z \nless_{L} x$, so $\operatorname{cl}(z)=\operatorname{cl}(x)$, as required.

We now define a total order $\prec$ on $X$, which will have the properties that

(1) if $x<_{L} y$ then $y \prec x$, and

(2) if $z \prec y \prec x$ and $z \in[x]_{\perp}$ then $y \in[x]_{\perp}$.

To begin with let

$$
B_{0}=\left\{Y \in L_{X} \mid Y=\operatorname{cl}(x) \text { where } x \text { is } L \text {-minimal }\right\} .
$$

Suppose that $B_{0}$ has $k$ elements and choose an ordering $Y_{1}<\cdots<Y_{k}$ of these elements. If $i \neq j$ then $Y_{i} \cap Y_{j} \in L$ and from the remark at the beginning of this section and the fact that the $Y_{i}$ 's are $L$-minimal it follows that $Y_{i} \cap Y_{j}=\emptyset$. Therefore we may define the ordering $\prec$ on $\bigcup_{i=1}^{k} Y_{i}$ in such a way that if $x_{i} \in Y_{i}$ and $x_{j} \in Y_{j}$ and $Y_{i}<Y_{j}$ then $x_{j} \prec x_{i}$ : merely by choosing an ordering for elements of each $Y_{i}$.

We recursively define sets $B_{i}$ of elements of $L_{X}$ for $i \geq 0$ as follows. Assume that we have defined sets $B_{0}, \ldots, B_{i}$, set $U_{i}=\bigcup_{j=0}^{i} B_{j}$ and define $X_{i}=\{u \in X \mid$ $u \in Y$ for some $\left.Y \in U_{i}\right\}$. If $U_{i} \neq L_{X}$ define $B_{i+1}$ by

$$
B_{i+1}=\left\{Y=\operatorname{cl}(x) \in L_{X} \mid Y \notin U_{i} \text { and } y<_{L} x \text { implies that } \operatorname{cl}(y) \in U_{i}\right\} .
$$

If $U_{i} \neq L_{X}$ then $X_{i} \neq X$ and $B_{i+1} \neq \emptyset$. We assume inductively that we have ordered the set $X_{i}$ in such a way that if $0 \leq a<b \leq i$ then $x_{a} \in Y_{a}$ where $Y_{a} \in B_{a}$ and $x_{b} \in Y_{b}$ where $Y_{b} \in B_{b}$ implies that $x_{b} \prec x_{a}$. From Proposition 2.7, if $Y=\operatorname{cl}(x) \in B_{i+1}$ then

$$
[x]_{\perp}=Y \backslash\left\{u \in \operatorname{cl}(y): y<_{L} x\right\}=Y \backslash\left\{u \in X_{i}\right\} .
$$

Therefore we have defined $\prec$ on the set $Y \backslash[x]_{\perp}$. Moreover, if $Y_{1} \neq Y_{2}$ and $Y_{1}, Y_{2} \in$ $B_{i+1}$ then $Y_{1} \cap Y_{2} \in L$, so $z \in Y_{1} \cap Y_{2}$ implies $\operatorname{cl}(z) \subseteq Y_{1} \cap Y_{2}$. As $Y_{1} \neq Y_{2}$ this implies that $\mathrm{cl}(z)$ is strictly contained in $Y_{i}, i=1$, 2. If $Y_{i}=\operatorname{cl}\left(x_{i}\right)$ then $z<_{L} x_{i}$ and so $z \notin\left[x_{i}\right]_{\perp}, i=1,2$. That is, $\left[x_{1}\right]_{\perp} \cap\left[x_{2}\right]_{\perp}=\emptyset$. Now choose an ordering on the set of elements of $B_{i+1}: Z_{1}<\cdots<Z_{k}$ say, where $Z_{j}=\operatorname{cl}\left(x_{j}\right)$. Then $Z_{j} \backslash\left[x_{j}\right]_{\perp} \subseteq X_{i}, j=1, \ldots, k$. We can extend the total order $\prec$ on $X_{i}$ to

$$
X_{i+1}=X_{i} \cup \bigcup_{j=1}^{k} Z_{j}=X_{i} \cup \bigcup_{j=1}^{k}\left[x_{j}\right]_{\perp}
$$


as follows. Assume the order has already been extended to $X_{i} \bigcup_{j=1}^{s-1}\left[x_{j}\right]_{\perp}$. Extend the order further by choosing the ordering $\prec$ on the elements of $\left[x_{s}\right]_{\perp}$ and then setting its greatest element less than the least element of $X_{i} \bigcup_{j=1}^{s-1}\left[x_{j}\right]_{\perp}$. At the final stage $s=k$ and the order on $X_{i}$ is extended to $X_{i+1}$. We continue until $U_{i}=L_{X}$, at which point $X=X_{i}$ and we have the required total order on $X$. Note that, by construction, if $x, y \in X$ and $x<_{L} y$ then $y \prec x$. Also, if $x \prec y \prec z$ and $[z]_{\perp}=[x]_{\perp}$ then $[y]_{\perp}=[x]_{\perp}$. Thus (1) and (2) above hold. If $\operatorname{cl}(x)$ belongs to $B_{i}$ we shall say that $x, \operatorname{cl}(x)$ and $[x]_{\perp}$ have height $i$ and write $h(x)=h(\operatorname{cl}(x))=h\left([x]_{\perp}\right)=i$.

2.5. A matrix representation of $\operatorname{St}(L)$. Suppose that $X=\left\{x_{1}, \ldots, x_{k}\right\}$ with $x_{1} \prec \cdots \prec x_{k}$. If $x \in X$ and $\phi \in \operatorname{St}(L)$ then we have $x^{\phi} \in G(\operatorname{cl}(x))$. If $\operatorname{cl}(x)=\left\{y_{1}, \ldots, y_{r}\right\}$, where $x=y_{1}$ say, then as $G(\operatorname{cl}(x))$ is a free Abelian group we may write

$$
x^{\phi}=y_{1}^{b_{1}} \ldots y_{r}^{b_{r}} .
$$

Setting $a_{j}=0$ if $x_{j} \notin \mathrm{cl}(x)$, and $a_{j}=b_{i}$ if $x_{j}=y_{i}$ for some $i$, we can write

$$
x^{\phi}=x_{1}^{a_{1}} \ldots x_{k}^{a_{k}} .
$$

As this holds for all $x \in X$ we have

$$
x_{1}^{\phi}=x_{1}^{a_{1,1}} \ldots x_{k}^{a_{1, k}}, \ldots, x_{k}^{\phi}=x_{1}^{a_{k, 1}} \ldots x_{k}^{a_{k, k}} .
$$

Assume now that $Y \in L$. Then $Y=\bigcup_{y \in Y} \operatorname{cl}(y)$ and for $1 \leq i \leq k$, either $\left[x_{i}\right]_{\perp} \subseteq Y$ or $\left[x_{i}\right]_{\perp} \cap Y=\emptyset$. Let $I=\left\{i \mid 1 \leq i \leq k\right.$ and $\left.\left[x_{i}\right]_{\perp} \subseteq Y\right\}$. Then, from Proposition 2.7, it follows that $Y=\bigcup_{i \in I}\left[x_{i}\right]_{\perp}$. Moreover for all $i$ such that $x_{i} \in Y$ we have

$$
x_{i}^{\phi}=\prod_{j \in I} x_{j}^{a_{i, j}} .
$$

We denote the restriction $\left.\phi\right|_{G(Y)}$ of $\phi$ to $G(Y)$ by $\phi_{Y}$ for any subset $Y$ of $X$ and $\phi \in \operatorname{St}(L)$. We shall also write $\phi_{x}$ instead of $\phi_{\operatorname{cl}(x)}$ for $x \in X$.

Definition 2.8. In the above notation, given $Y \in L$ we define the matrix corresponding to the restriction $\phi_{Y}$ of $\phi \in \operatorname{St}(L)$ to $G(Y)$ to be $\left[\phi_{Y}\right]=\left(a_{i, j}\right)_{i, j \in I}$. If $Y=X$ we write $[\phi]$ for $\left[\phi_{X}\right]$.

Definition 2.9. Let $Y=\left\{y_{1}, \ldots, y_{r}\right\} \in L$ with $y_{1} \prec \cdots \prec y_{r}$ and let $Z$ be a subset of $Y$. Let $I=\left\{i \mid 1 \leq i \leq r, y_{i} \in Z\right\}$. Given $A=\left(a_{i, j}\right) \in \mathrm{GL}(r, \mathbb{Z})$ we define the $Z$-minor of $A$ to be the matrix $M(A, Y, Z)=\left(a_{i, j}\right)_{i, j \in I}$. If $Y=X$ we write $M(A, Z)$ for $M(A, X, Z)$.

The $Z$-minor of a matrix $A$ is therefore the matrix obtained from $A$ by deleting the $i$ th row and column for all $i$ such that $y_{i} \in Y \backslash Z$. From these definitions we have the following lemma. 
Lemma 2.10. Let $\phi \in \operatorname{St}(L)$ and $Y \in L$ then $\left[\phi_{Y}\right]=M([\phi], Y)$. If $Z \subseteq Y \subseteq W$ are elements of $L$ then $M(A, W, Z)=M(M(A, W, Y), Y, Z)$.

For $x \in X$ we say that $y \in[x]_{\perp}$ is the minimal element of $[x]_{\perp}$ if $y \prec z$ for all $z \in[x]_{\perp}$. Let $X^{\text {min }}=\left\{x \in X \mid x\right.$ is the minimal element of $\left.[x]_{\perp}\right\}$. Then $X=\bigcup_{x \in X}[x]_{\perp}=\bigsqcup_{x \in X^{\min }}[x]_{\perp}$. We extend this notation to arbitrary $Y \in L$ by defining $Y^{\min }=X^{\min } \cap Y$; so $Y=\bigsqcup_{y \in Y_{\min }}[y]_{\perp}$.

2.6. Sets of matrices corresponding to closed sets. We define a set of integer valued upper block-triangular matrices corresponding to a closed set. Let $Y \in L$ and write $Y^{\text {min }}=\left\{v_{1}, \ldots, v_{m}\right\}$, where $v_{1} \prec \cdots \prec v_{m}$. Assume further that $Y=\left\{u_{1}, \ldots, u_{r}\right\}$, where $u_{1} \prec \cdots \prec u_{r}$. The set $S_{Y}$ is defined to the set of $r \times r$ integer valued matrices $A=\left(a_{i, j}\right)$ such that the following conditions hold.

(1) $A$ has $m$ diagonal blocks $A_{1}, \ldots, A_{m}$, such that $A_{i} \in \mathrm{GL}\left(\left|\left[v_{i}\right]_{\perp}\right|, \mathbb{Z}\right)$.

(2) If $i>j$ and $a_{i, j}$ is not part of a diagonal block then $a_{i, j}=0$.

(3) If $i<j$ and $a_{i, j}$ is not part of a diagonal block then $a_{i, j}=0$ unless $u_{j}<_{L} u_{i}$, in which case $a_{i, j}$ may be any element of $\mathbb{Z}$.

The first two of these conditions imply that $A$ is an upper block-triangular matrix. Suppose that $A \in S_{Y}$ and has diagonal blocks $A_{1}, \ldots, A_{m}$. Define the matrix $B$ to be the block-diagonal matrix with diagonal blocks $A_{1}^{-1}, \ldots, A_{m}^{-1}$. Then $A B$ is a unipotent matrix and it follows that $A \in \mathrm{GL}(r, \mathbb{Z})$. Therefore $S_{Y}$ is a subset of $\mathrm{GL}(r, \mathbb{Z})$.

Lemma 2.11. (1) Elements of $S_{Y}$ are upper block-triangular elements of $\mathrm{GL}(r, \mathbb{Z})$.

(2) If $\phi$ is an element of $J, V_{\perp}$ or $\operatorname{Tr}_{\perp}$ then $[\phi] \in S_{X}$.

(3) The set $S_{Y}$ (with matrix multiplication) is a monoid for all $Y \in L$.

Proof. If $\phi \in J$ then $[\phi]$ is a diagonal matrix with diagonal entries \pm 1 , so belongs to $S_{X}$. If $\phi \in V_{\perp}$ then $\phi=\prod_{[x]_{\perp} \in N_{2}^{\prime}} \phi_{x}^{\varepsilon_{x}}$ for some automorphisms $\phi_{x} \in \operatorname{Aut}\left(G[x]_{\perp}\right)$. Hence $[\phi]$ is block diagonal, with a blocks of dimension $\left|[x]_{\perp}\right|$ for each $[x]_{\perp} \in N_{2}^{\prime}$ and of dimension 1 for all $x \in N_{1}$. It follows that $[\phi] \in S_{X}$. Finally let $\phi=\operatorname{tr}_{x, y} \in \operatorname{Tr}_{\perp}$. Then $\operatorname{cl}(y)<\operatorname{cl}(x)$ so $x \prec y$ and if $x=x_{i}$ and $y=x_{j}$ then $[\phi]$ is the matrix with 1 's on the leading diagonal, $a_{i, j}=1$ and 0 's elsewhere. As $x_{i} \prec x_{j}$ we have $i<j$ and, as $x_{j}<_{L} x_{i}, a_{i, j}$ is not in a diagonal block so this matrix belongs to $S_{X}$. Thus statement (2) holds.

To prove statement (3) assume that $Y=\left\{u_{1}, \ldots, u_{r}\right\}$ where $u_{1} \prec \cdots \prec u_{r}$. Let $A, B \in S_{Y}, A=\left(a_{i, j}\right), B=\left(b_{i, j}\right)$ and let $A B=C=\left(c_{i, j}\right) \in \operatorname{GL}(r, \mathbb{Z})$. Then $c_{i, j}=\sum_{k=1}^{r} a_{i, k} b_{k, j}$. Suppose that $A$ has diagonal blocks $A_{1}, \ldots, A_{m}$. Let $A_{D}$ be the block-diagonal matrix with diagonal blocks $A_{1}, \ldots, A_{m}$ and 0 's elsewhere. Let $A_{N}=A-A_{D}$. Define $B_{D}$ and $B_{N}$ similarly, so $B_{D}$ is block diagonal and $B=B_{D}+B_{N}$. Then $C=A_{D} B_{D}+A_{D} B_{N}+A_{N}\left(B_{D}+B_{N}\right)$. Therefore $C$ is upper block-triangular with diagonal blocks $A_{1} B_{1}, \ldots, A_{m} B_{m}$ and $A_{i} B_{i} \in \mathrm{GL}\left(\left|A_{i}\right|, \mathbb{Z}\right)$. 
Suppose that $i<j$ and $c_{i, j}$ does not belong to a diagonal block. If $i$ and $k$ are such that $u_{k} \not_{L} u_{i}$ then $a_{i, k}=0$ and if $k, j$ are such that $u_{j} \not_{L} u_{k}$ then $b_{k, j}=0$. Hence $a_{i, k} b_{k, j} \neq 0$ implies that $u_{k} \leq_{L} u_{i}$ and $u_{j} \leq_{L} u_{k}$. If $u_{i}==_{L} u_{j}$ then $c_{i, j}$ belongs to a diagonal block, a contradiction. Hence $u_{j}<_{L} u_{i}$. Thus $c_{i, j} \neq 0$ implies $u_{j}<_{L} u_{i}$ and so $C \in S_{Y}$. As the identity matrix is in $S_{Y}$ it follows that $S_{Y}$ is a monoid.

We are now ready to prove Theorem 2.4, which is the second statement of the following result.

Theorem 2.12. The map $\pi: \operatorname{St}(L) \rightarrow \mathrm{GL}(|X|, \mathbb{Z})$ given by $\phi \mapsto[\phi]$ is an injective homomorphism with image $S_{X}$. In particular $S_{X}$ is a group. Moreover the group $\operatorname{St}(L)$ is generated by the elements of $J, V_{\perp}$ and $\operatorname{Tr}_{\perp}$, and is an arithmetic group.

Proof. We shall first show that $\pi$ is an injective group homomorphism, from $\operatorname{St}(L)$ to $\mathrm{GL}(|X|, \mathbb{Z})$. Assume that $|X|=k$, let $\phi, \psi \in \operatorname{St}(L)$ and let $[\phi]=\left(a_{i, j}\right)$ and $[\psi]=\left(b_{i, j}\right)$. In the notation of Section 2.4 for $x_{i} \in X$ we have $x_{i}^{\phi \psi}=$ $\left(x_{1}^{a_{i, 1}}\right)^{\psi} \ldots\left(x_{k}^{a_{i, k}}\right)^{\psi}=\prod_{r=1}^{k}\left(x_{1}^{b_{r, 1}} \ldots x_{k}^{b_{r, k}}\right)^{a_{i, r}}=\prod_{j=1}^{k} x_{j}^{c_{i, j}}$, where $c_{i, j}=$ $\sum_{r=1}^{k} a_{i, r} b_{r, j}$. Hence $[\phi \psi]=\left(c_{i, j}\right)=[\phi][\psi]$. Therefore $\pi$ is a homomorphism and it is immediate from the definition that $\pi$ is injective.

Let $T$ denote the subgroup of $\operatorname{St}(L)$ generated by $J, V_{\perp}$ and $\operatorname{Tr}_{\perp}$ and recall that $V$ is the subgroup of $\operatorname{St}(L)$ generated by $J$ and $V_{\perp}$. From Lemma 2.11 it follows that $[\phi] \in S_{X}$ for all $\phi \in T$. Therefore once we have proved the final statement of the Lemma it will also follow that the image of $\pi$ is contained in $S_{X}$. The proof of the final statement will be broken into three cases and in each case we shall also verify that $\pi$ maps surjectively onto $S_{X}$.

Let $\phi \in \operatorname{St}(L)$ and $x \in X$ and suppose that $\operatorname{cl}(x)=\left\{y_{1}, \ldots, y_{r}\right\}$. Then we can express $x^{\phi}$ as in (2.2). Assume further that $[x]_{\perp}=\left\{y_{1}, \ldots, y_{s}\right\}$, where $s \leq r$. In the notation of (2.2), if $b_{j}=0$ for all $j>s$, then $x^{\phi} \in G[x]_{\perp}$. Suppose this holds for all $x \in[x]_{\perp}$; so $\phi_{[x]_{\perp}} \in \operatorname{Aut}\left(G[x]_{\perp}\right)$. In this case we call $\phi$ a block-diagonal automorphism.

Case 1. Let $\phi \in \operatorname{St}(L)$ be a block-diagonal automorphism and let $x \in X$. If $x \in N_{2}$ then $[x]_{\perp} \in N_{2}^{\prime}$ and $\phi_{[x]_{\perp}}^{\varepsilon_{x}} \in \operatorname{Aut}\left(G[x]_{\perp}\right)^{\varepsilon_{x}} \subseteq V_{\perp}$. If $x \notin N_{2}$ then $[x]_{\perp}=\{x\}$ and $\phi_{[x]_{\perp}}^{\varepsilon_{x}} \in J$. In either case $\phi_{[x]_{\perp}}^{\varepsilon_{x}} \in V$ and, as the same is true of all $x \in X$,

$$
\phi=\prod_{[x]_{\perp} \in N_{2}^{\prime}} \phi_{[x]_{\perp}}^{\varepsilon_{x}} \cdot \prod_{x \in N_{1}} \phi_{[x]_{\perp}}^{\varepsilon_{x}} \subseteq V \subseteq T .
$$

Now let $X=\left\{x_{1}, \ldots, x_{k}\right\}$, where $x_{1} \prec \cdots \prec x_{k}$ and write $[\phi]=\left(a_{i, j}\right)$. Let $X^{\mathrm{min}}=\left\{x_{i_{1}}, \ldots, x_{i_{m}}\right\}$ for some $m \geq 1$, with $x_{i_{1}} \prec \cdots \prec x_{i_{m}}$. In this terminology what we have shown is the following.

If $a_{i, j}=0$ for all $i, j$ such that $x_{i} \in\left[x_{i_{n}}\right]_{\perp}, x_{j} \notin\left[x_{i_{n}}\right]_{\perp}$, then $\phi_{\left[x_{i_{n}}\right]_{\perp}}^{\varepsilon_{i_{n}}} \in V$; and if this holds for $n=1, \ldots, m$, then $\phi=\prod_{n=1}^{m} \phi_{\left[x_{i_{n}}\right]_{\perp}}^{\varepsilon_{i_{n}}} \in V \subseteq T$. 
That is, if the above holds then $\phi \in T$, and so $[\phi] \in S_{X}$.

On the other hand, let $A \in S_{X}$ be a block-diagonal matrix. Then $A$ has diagonal blocks $A_{n} \in \mathrm{GL}\left(\left|\left[x_{i_{n}}\right]_{\perp}\right|, \mathbb{Z}\right)$ for $n=1, \ldots, m$. Here $A_{n}$ determines an automorphism, $\phi_{n}$ say, of $G\left[x_{i_{n}}\right]_{\perp}$, and $\phi=\prod_{n=1}^{m} \phi_{n}^{\varepsilon_{x_{i n}}} \in V$. Moreover $[\phi]=A$; so all block-diagonal matrices in $S_{X}$ are in the image of $\pi$.

Case 2. Let $\phi \in \operatorname{St}(L)$ and $A=[\phi]$. Write $A=A_{D}+A_{N}$ as in the proof of Lemma 2.11. In this case we assume that $A_{D}$ is the identity matrix. This means that $x_{i}^{\phi}=x_{i} w_{i}$, where $w_{i} \in G(Y)$ for some $Y \subseteq \operatorname{cl}(x) \backslash\left[x_{i}\right]_{\perp}$ for $i=1, \ldots, k$. Define $r=r(\phi)$ to be the maximal integer such that $x_{r} \in\left[x_{i_{n}}\right]_{\perp}$ for some $n$, and there exists $x_{j}$ such that $x_{j} \notin\left[x_{i_{n}}\right]_{\perp}$ but $a_{r, j} \neq 0$. Let $j=c=c(\phi)$ be maximal with this property. (The argument of case 1 covers the case $r=0$.) As $a_{r, c} \neq 0$ we have $w_{r}=u x_{c}^{a_{r, c}}$ for some $u \in G(Z)$, where $x_{r} \notin Z$ and, since $A_{D}$ is the identity $w_{s} \in G\left(Z_{s}\right)$, where $x_{r} \notin Z_{s}$ for all $s>r$.

As $\phi \in \operatorname{St}(L)$ we have $x_{r}^{\phi} \in \operatorname{cl}\left(x_{r}\right)$, thus $x_{c} \in \operatorname{cl}\left(x_{r}\right)$, which implies that $\operatorname{cl}\left(x_{c}\right) \subseteq \operatorname{cl}\left(x_{r}\right)$. Since $x_{c} \notin\left[x_{r}\right]_{\perp}$ it follows that $\operatorname{cl}\left(x_{c}\right) \neq \operatorname{cl}\left(x_{r}\right)$. Hence $\operatorname{tr}_{x_{r}, x_{c}} \in$ $\operatorname{Tr}_{\perp} \subseteq \operatorname{St}(L)$. Let $\phi_{1}=\left(\operatorname{tr}_{x_{r}, x_{c}}\right)^{-a_{r, c}} \in T$. Then $x_{r}^{\phi_{1}}=x_{r} x_{c}^{-a_{r, c}}$ and $x_{l}^{\phi_{1}}=x_{l}$ for all $l \neq r$. Let $\phi_{0}=\phi \phi_{1}$; so $\phi_{0} \in \operatorname{St}(L)$. We have

$$
x_{r}^{\phi_{0}}=\left(x_{r} u x_{c}^{a_{r, c}}\right)^{\phi_{1}}=x_{r} u \quad \text { and } \quad x_{s}^{\phi_{0}}=\left(x_{s} w_{s}\right)^{\phi_{1}}=x_{s} w_{s}=x_{s}^{\phi}
$$

for $s>r$. If $s<r$ then

$$
x_{s}^{\phi_{0}}=x_{s} w_{s}^{\phi_{1}}= \begin{cases}x_{s} w_{s} x_{c}^{a_{r, c}}=x_{s}^{\phi} x_{c}^{a_{r, c}} & \text { if } x_{r} \text { occurs in } w_{s}, \\ x_{s} w_{s}=x_{s}^{\phi} & \text { otherwise. }\end{cases}
$$

Therefore all diagonal blocks of $\left[\phi_{0}\right]$ are the identity matrix and either $r\left(\left[\phi_{0}\right]\right)<r$ or $c\left(\left[\phi_{0}\right]\right)<c$. We may then assume inductively that $\left[\phi_{0}\right] \in S_{X}$ and $\phi_{0} \in T$ : so $\phi \in T$. Now define $E$ to be the matrix which has zero in every position except row $r$ column $c$, which is equal to $a_{r, c}$. Then $\left[\phi_{1}^{ \pm 1}\right]=I \mp E \in S_{X}$ and from Lemma 2.11 it follows that $[\phi]=\left[\phi_{0}\right]\left[\phi_{1}^{-1}\right] \in S_{X}$. By induction it follows that for all $\phi$ such that $[\phi]=A=A_{D}+A_{N}$, with $A_{D}$ the identity, we have $\phi \in T$ and $\phi^{\pi} \in S_{X}$. The same argument shows that if $A \in S_{X}$ and $A=A_{D}+A_{N}$, with $A_{D}$ the identity, then $A=\phi^{\pi}$ for some $\phi \in T$.

Case 3. In the general case let $\phi \in \operatorname{St}(L)$ and write $[\phi]=A=A_{D}+A_{N}$ as before. Let $B=A_{D}^{-1}$. Then from case $1, B^{ \pm 1} \in S_{X}$ and $B=\left[\sigma_{B}\right]$ for some $\sigma_{B} \in T$. Let $\zeta=\phi \sigma_{B}$. Then all diagonal blocks of $[\zeta]$ are the identity, so $\zeta \in T$ and $[\zeta] \in S_{X}$, from case 2 . Therefore $\phi \in T$ and $[\phi] \in S_{X}$. If we begin this argument with an arbitrary element $A$ of $S_{X}$ instead of an element of $\operatorname{St}(L)$ it shows again that $A \in T^{\pi}$ and $A \in S_{X}$.

We now show that the group $\operatorname{St}(L)$ is an arithmetic group. Let $K$ be the subgroup of GL $(|X|, \mathbb{C})$ satisfying conditions (1), (2) and (3) in the definition of $S_{X}$ above. Then $K$ is a $\mathbb{Q}$-defined linear algebraic group. As $S_{X}=K \cap G L(|X|, \mathbb{Z})$ it now follows that $\operatorname{St}(L)$ is arithmetic. 
Combining the final statement of the theorem with Proposition 2.6 we obtain the following corollary.

Corollary 2.13. $\operatorname{St}\left(L^{\max }\right)$ is an arithmetic group.

Proof. From the proof of Proposition 2.6 it follows that $\operatorname{St}(L)$ is a finite index subgroup of $\operatorname{St}\left(L^{\max }\right)$. Also the proof of of Theorem 2.12 goes through to show that $\operatorname{St}\left(L^{\max }\right)$ is isomorphic to a subgroup $S^{\max }$ of $\operatorname{GL}(|X|, \mathbb{Z})$. To see this for each $x \in X$, let $M_{x}=\left\{z \in X \mid z\right.$ is $L$-maximal and $\left.x<_{L} z\right\}$. Then $G\left(M_{x}\right)$ is Abelian and contains $x^{\phi}$ for all $\phi \in \operatorname{St}\left(L^{\max }\right)$. Let $\phi \in \operatorname{St}\left(L^{\max }\right)$ and, as at the beginning of Section 2.5, write $x^{\phi}=x_{1}^{a_{1}} \ldots x_{k}^{a_{k}}$, where $a_{j} \neq 0$ only if $x_{j} \in M_{x}$. As before this allows us to associate an integer valued matrix $[\phi]$ to $\phi$. The proof that the map $\phi \mapsto[\phi]$ is a monomorphism from $\operatorname{St}\left(L^{\max }\right)$ into $\mathrm{GL}(|X|, \mathbb{Z})$ is exactly the same as the first paragraph of the proof of Theorem 2.12. Thus $\operatorname{St}\left(L^{\max }\right)$ is isomorphic to its image $S^{\max } \subseteq \mathrm{GL}(|X|, \mathbb{Z})$. Moreover this monomorphism restricts to $\operatorname{St}(L)$ to give the map $\pi$ and so $S_{X}$ is a finite index subgroup of $S^{\max }$.

Keeping the notation of the proof of the previous theorem we have $S_{X}=K \cap$ $\operatorname{GL}(|X|, \mathbb{C})$. Now choose a transversal $a_{1}, \ldots, a_{s}$ for cosets of $S_{X}$ in $S^{\max }$. Then $g \in S^{\max }$ if and only if $g a_{r}^{-1} \in S_{X} \subseteq K$ for some $r$. As $S^{\max } \in \mathrm{GL}(|X|, \mathbb{Z})$ so $a_{r}^{-1} \in \mathrm{GL}(|X|, \mathbb{Z})$ for all $r$. Hence the condition that an element $h \in \mathrm{GL}(|X|, \mathbb{C})$ satisfies $h=g a_{r}^{-1}$ for some $g$, can be expressed using $|X|^{2}$ polynomials with integer coefficients (namely the entries of the matrix $a_{r}^{-1}$ ). Set $p=|X|$ and let these polynomials be $m_{r, 1,1}, \ldots, m_{r, p, p}$. (Thus if $h=g a_{r}^{-1}=\left(h_{i j}\right)$ then substitution of entries of $g$ for variables of the $m_{r, i, j}$ gives $h_{i j}=m_{r, i, j}(g)$ for all $i, j$.) Suppose that the algebraic variety $K$ is defined by polynomials $f_{1}, \ldots, f_{l}$. Then $g \in K a_{r}$ if and only if $g$ satisfies the polynomial equations $f_{i}\left(m_{r, 1,1}, \ldots, m_{r, p, p}\right), i=1, \ldots, l$. As $f_{i}$ and $m_{r, i, j}$ are polynomials with integer coefficients, this implies that $K a_{r}$ is a $\mathbb{Q}$-defined affine algebraic variety. Thus $\bigcup_{r=1}^{s} K a_{r}$ is a variety and

$$
\left(\bigcup_{r=1}^{s} K a_{r}\right) \cap \mathrm{GL}(|X|, \mathbb{Z})=\bigcup_{r=1}^{s}(K \cap \mathrm{GL}(|X|, \mathbb{Z})) a_{r}=\bigcup_{r=1}^{s} S_{X} a_{r}=S^{\max },
$$

so $\operatorname{St}\left(L^{\max }\right)$ is an arithmetic group.

In the previous theorem we restricted attention to the entire group $\operatorname{St}(L)$ and its isomorphic image $S_{X}$. However, we shall now show that the set $S_{Y}$ is a group for all closed sets $Y$ in $L$, and in fact all these groups are arithmetic. By defining appropriate maps corresponding to inclusion, as follows, it can be seen that the lattice $L$ maps contravariantly to a sublattice of the lattice of subgroups of $\operatorname{Aut}(G)$. If $Y, Z \in L$ with $Y \subseteq Z$ then $M(A, Y, Z) \in \mathrm{GL}(|Z|, \mathbb{Z})$ and so $\rho(Y, Z): A \mapsto M(A, Y, Z)$ is a map from $S_{Y}$ to $\mathrm{GL}(|Z|, \mathbb{Z})$.

Lemma 2.14. Let $Z, Y \in L$ with $Z \subseteq Y$. The set $S_{Y}$ is an arithmetic group and the map $\rho(Y, Z)$ is a surjective homomorphism from $S_{Y}$ to $S_{Z}$. There is an injective 
homomorphism $\varepsilon(Z, Y)$ from $S_{Z}$ to $S_{Y}$ such that $\varepsilon(Z, Y) \rho(Y, Z)$ is the identity on $S_{Z}$.

Proof. We show that $\rho(Y, Z)$ is an surjective monoid homomorphism for all $Z \subseteq$ $Y \in L$. Since $S_{X}$ is a group it will then follow that $S_{Y}$ is a group for all $Y \in L$. The proof that $S_{Y}$ is arithmetic is then the same as for $S_{X}$, replacing $X$ by $Y$ throughout. Let $Y^{\text {min }}=\left\{v_{1}, \ldots, v_{m}\right\}$, where $v_{1} \prec \cdots \prec v_{m}$. Also let $Y=\left\{u_{1}, \ldots, u_{r}\right\}$, where $u_{1} \prec \cdots \prec u_{r}$ and let $I=\left\{i \mid 1 \leq i \leq r\right.$ and $\left.u_{i} \in Z\right\}$. Let $A=\left(a_{i, j}\right) \in S_{Y}$ and suppose that $A$ has diagonal blocks $A_{1}, \ldots, A_{m}$. As $A$ is upper block-triangular it follows from the definition that $M(A, Y, Z)$ is upper block-triangular. If $v_{i} \in Z$ then $\left[v_{i}\right]_{\perp} \subseteq Z$ and the diagonal block containing $A_{i}$ is unaffected in the transformation of $A$ to $M(A, Y, Z)$. On the other hand if $v_{i} \notin Z$ then the diagonal block $A_{i}$ is deleted in forming $M(A, Y, Z)$. As $Z^{\mathrm{min}}=Y^{\mathrm{min}} \cap X$, the diagonal blocks of $M(A, Y, Z)$ satisfy condition (1) of the definition of $S_{Z}$.

It remains to verify condition (3). Suppose that $i, j \in I$ and that $a_{i, j} \neq 0$ and $a_{i, j}$ does not belong to a diagonal block of $M(A, Y, Z)$. From the above, $a_{i, j}$ does not belong to a diagonal block of $A$, and since $A$ is upper block-triangular and satisfies condition (3), $i<j$ and $u_{j}<_{L} u_{i}$. Then the same holds for $M(A, Y, Z)$, as required. Therefore $\rho(Y, Z)$ maps $S_{Y}$ into $S_{Z}$.

To see that $\rho(Y, Z)$ is a homomorphism let $A=\left(a_{i, j}\right)$ and $B=\left(b_{i, j}\right)$ be elements of $S_{Y}$ and let $C=\left(c_{i, j}\right)=A B$. From Lemma 2.11, we have $C \in S_{Y}$. Suppose that $i, j \in I$ and that $a_{i, k} b_{k, j} \neq 0$ for some $k$. Then $u_{k} \leq_{L} u_{i}$ and $u_{i} \in Z$. Hence $u_{k} \in$ $\operatorname{cl}\left(u_{k}\right) \subseteq \operatorname{cl}\left(u_{i}\right) \subseteq Z$. Therefore $i, j, k \in I$ and $c_{i, j}=\sum_{k \in I} a_{i, k} b_{k, j}$. It follows that $M(A B, Y, Z)=M(A, Y, Z) M(B, Y, Z)$, so $\rho(Y, Z)$ is a homomorphism.

To construct $\varepsilon(Z, Y)$ note that if $P \in S_{Z}$ then we may write $P=\left(p_{i, j}\right)_{i, j \in I}$, by expressing $Z$ as a subset of $\left\{u_{1}, \ldots, u_{r}\right\}$. Then let the diagonal blocks of $P$ be $P_{i}$, where $i \in I$. With this notation define an $r \times r$ integer matrix $A$ by first of all setting $a_{i, j}=p_{i, j}$ for $i, j \in I$, then setting $a_{i, i}=1$ for $i \notin I$, and finally setting $a_{i, j}=0$ for all other $i, j$. Then $A$ is upper block-triangular and has blocks $A_{1}$, $\ldots, A_{m}$, where $A_{i}=P_{i}$ if $i \in I$, and $A_{i}$ is the identity matrix in $\mathrm{GL}\left(\left|\left[v_{i}\right]_{\perp}\right|, \mathbb{Z}\right)$ otherwise. As $P$ satisfies condition (3) then so does $A$. Hence $A$ belongs to $S_{Y}$. Define $M(P, Z, Y)=A$ (where $Z \subseteq Y$ ) and $P^{\varepsilon(Y, Z)}=M(P, Z, Y)$ for all $P \in S_{Z}$. By definition, $P^{\varepsilon(Y, Z) \rho(Y, Z)}=P$ for all $P \in S_{Z}$, so $\varepsilon(Y, Z)$ is injective and $\rho(Y, Z)$ is surjective. Moreover, from the definition, $\varepsilon(Y, Z)$ is a homomorphism.

2.7. Restriction to closed sets. Here we consider the restriction of automorphisms in $\operatorname{St}(L)$ to subgroups $G(Y)$, where $Y$ is in $L$. Given $Y \in L$ let us define $L(Y)=$ $\{Z \in L \mid Z \subseteq Y\}$. Note that $L(Y)$ is not in general the same as $L(\Gamma(Y))$, the set of closed sets of the full subgraph $\Gamma(Y)$ of $\Gamma$ generated by $Y$; although $L \Gamma(Y)) \subseteq L(Y)$. We define $\operatorname{St}_{Y}(L)=\left\{\phi_{Y} \mid \phi \in L\right\}$. Then $\operatorname{St}_{Y}(L)$ is a subgroup of $\operatorname{Aut}(G(Y))$ and is contained in the subgroup of stabilisers, in $\operatorname{Aut}(G(Y))$, of $L(Y)$. 
Lemma 2.15. The map $\rho_{Y}: \operatorname{St}(L) \rightarrow \operatorname{St}_{Y}(L)$ given by $\phi \mapsto \phi_{Y}$ is a surjective homomorphism. The map $\pi_{Y}: \mathrm{St}_{Y}(L) \rightarrow S_{Y}$ given by $\phi_{Y} \mapsto\left[\phi_{Y}\right]$ is an isomorphism, so $\mathrm{St}_{Y}(L)$ is arithmetic for all $Y \in L$. Moreover $\rho_{Y} \pi_{Y}=\pi_{X} \rho(X, Y)$.

Proof. Let $\phi, \psi \in \operatorname{St}(L)$. Then $x^{\phi} \in G(Y)$ for all $x \in Y$, so $x^{\phi \psi}=\left(x^{\phi}\right)^{\psi}=$ $\left(x^{\phi_{Y}}\right)^{\psi_{Y}}$ for all $x \in Y$. Hence $(\phi \psi)_{Y}=\phi_{Y} \psi_{Y}$ and $\rho_{Y}$ is a homomorphism; surjective onto its image which is, by definition, $\operatorname{St}_{Y}(L)$.

From Theorem 2.12 the map $\pi_{X}=\pi$ is an isomorphism from $\operatorname{St}(L)$ to $S_{X}$. From Lemma 2.14 the map $\rho(X, Y)$ is a surjective homomorphism from $S_{X}$ to $S_{Y}$. Let $\theta=\pi_{X} \rho(X, Y)$. An element $\phi \in \operatorname{St}(L)$ belongs to $\operatorname{ker}\left(\rho_{Y}\right)$ if $x^{\phi}=x$ for all $x \in Y$ : in which case $\left[\phi_{Y}\right]$ is the identity matrix of dimension $|Y|$. Hence the diagonal blocks of $\phi^{\pi_{X}}$ corresponding to $[x]_{\perp} \subseteq Y$ are identity matrices; and $\phi^{\theta}=I$, the $|Y|$-dimensional identity matrix. This shows that $\operatorname{ker}\left(\rho_{Y}\right) \subseteq \operatorname{ker}(\theta)$, so $\theta$ induces a homomorphism from $\operatorname{St}_{Y}(L)$ to $S_{Y}$. The image of $\phi_{Y}$ under this homomorphism is $\phi^{\theta}=[\phi]^{\rho(X, Y)}=M([\phi], Y)$ and from the definitions we have $\phi_{Y}^{\pi_{Y}}=M([\phi], Y)$. Therefore $\pi_{Y}$ is a homomorphism and $\rho_{Y} \pi_{Y}=\pi_{X} \rho(X, Y)$. As $\theta$ is surjective, so is $\pi_{Y}$. If $\phi_{Y}^{\pi_{Y}}=I$ then $x^{\phi_{Y}}=x$ for all $x \in Y$, so $\phi_{Y}$ is the identity on $G(Y)$ and $\pi_{Y}$ is injective.

If $Z \subseteq Y \in L$ and $\phi \in \operatorname{St}(L)$ we define $\rho_{Y, Z}$ to be the map sending $\phi \in \operatorname{St}_{Y}(L)$ to $\left.\phi\right|_{G(Z)} \in \operatorname{St}_{Z}(L)$.

Corollary 2.16. Let $Y, Z \in L$ with $Z \subseteq Y$. Then $\rho(X, Z)=\rho(X, Y) \rho(Y, Z)$ and $\rho_{Z}=\rho_{Y} \rho_{Y, Z}$. Moreover $\rho_{Y, Z}$ is surjective and $\pi_{Y} \rho(Y, Z)=\rho_{Y, Z} \pi_{Z}$.

Proof. This follows from Lemmas 2.10, 2.14 and 2.15.

The various maps we have defined are illustrated in the commutative diagram of Figure 1.

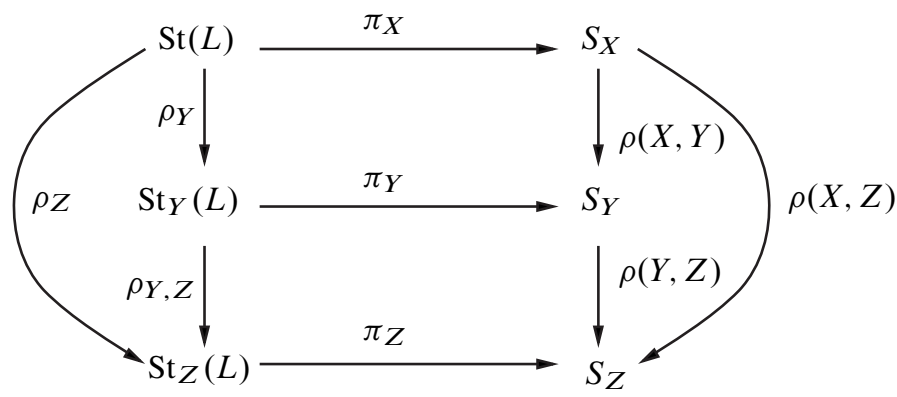

Figure 1. Maps defined on subgroups of $\operatorname{St}(L)$. 
2.8. The structure of $\mathbf{S t}^{\mathrm{conj}}(\boldsymbol{L})$. First we examine the structure of $\operatorname{St}_{Y}(L)$ and $S_{Y}$ for an arbitrary closed set $Y$.

Definition 2.17. Let $Y \in L$ and $Y=\bigcup_{i=1}^{m}\left[v_{i}\right]_{\perp}$, where $Y^{\min }=\left\{v_{1}, \ldots, v_{m}\right\}$ with $v_{1} \prec \cdots \prec v_{m}$. Denote by $D_{Y}$ the set of block diagonal elements of $S_{Y}$ with diagonal blocks $A_{1}, \ldots, A_{m}$ such that $A_{i} \in \mathrm{GL}\left(\left|\left[v_{i}\right]_{\perp}\right|, \mathbb{Z}\right)$. Denote by $U_{Y}$ the subset of $S_{Y}$ consisting of matrices for which $A_{i}$ is the identity matrix of GL $\left(\left|\left[v_{i}\right]_{\perp}\right|, \mathbb{Z}\right)$ for $i=1, \ldots, m$.

Lemma 2.18. Let $Y \in L$ and $Y=\bigcup_{i=1}^{m}\left[v_{i}\right]_{\perp}$, where $Y^{\min }=\left\{v_{1}, \ldots, v_{m}\right\}$ with $v_{1} \prec \cdots \prec v_{m}$. Both $U_{Y}$ and $D_{Y}$ are subgroups of $S_{Y}$ and $S_{Y}=U_{Y} \rtimes D_{Y}$. Moreover

$$
D_{Y}=\prod_{i=1}^{m} G\left[v_{i}\right]_{\perp} .
$$

Proof. Let $A \in S_{Y}$ with diagonal blocks $A_{1}, \ldots, A_{m}$ and define $A_{D}$ to be the blockdiagonal matrix with diagonal blocks $A_{1}, \ldots, A_{m}$, and let $d$ be the map sending $A$ to $A_{D}$. Then $\left(A^{d}\right)^{\pi_{Y}^{-1}}$ is clearly an element of $\operatorname{St}_{Y}(L)$ and so $A^{d} \in S_{Y}$. Hence $A^{d} \in D_{Y}$ and $d$ is a surjective map from $S_{Y}$ to $D_{Y}$. If $B$ is also in $S_{Y}$ and has diagonal blocks $B_{1}, \ldots, B_{m}$ then, as in the proof of Lemma 2.11, $A B$ has blocks $A_{1} B_{1}, \ldots, A_{m} B_{m}$, so $d$ is a homomorphism and $D_{Y}$ is a group. If $A \in S_{Y}$ then $A \in \operatorname{ker}(d)$ if and only if every diagonal block of $A$ is an identity matrix. Hence $\operatorname{ker}(d)=U_{Y}$. If $i$ is the inclusion of $D_{Y}$ in $S_{Y}$ then $i d$ is the identity map on $D_{Y}$ and so $S_{Y}=U_{Y} \rtimes D_{Y}$, as claimed. That $D_{Y}=\prod_{i=1}^{m} G\left[v_{i}\right]_{\perp}$ is immediate from the definitions.

In the case where $Y$ is the closure of a single element of $X$ we have the following corollary of the above results.

Corollary 2.19. (1) $\left[\phi_{x}\right] \in S_{x}$ for all $\phi \in \operatorname{St}(L)$.

(2) There are automorphisms $\phi_{x, s}$ and $\phi_{x, u}$ of $G(\mathrm{cl}(x))$ such that $\phi_{x}=\phi_{x, s} \phi_{x, u}$ and $\left[\phi_{x, s}\right]$ is the block-diagonal matrix with diagonal blocks $A_{1}, \ldots, A_{m}$ and $\left[\phi_{x, u}\right]$ is an upper unitriangular matrix (the unipotent part of $\left[\phi_{x}\right]$ ).

(3) Given $A \in S_{x}$ there exists $\psi \in \operatorname{St}(L)$ such that $\left[\psi_{x}\right]=A$.

(4) If $y<_{L} x$ then $\left[\phi_{y}\right]=M\left(\left[\phi_{x}\right], \operatorname{cl}(y)\right)$.

(5) The set $S_{x}$ is a group.

Proof. Assertions (1), (3) and (4) follow from Lemma 2.15. Statement (5) follows from Lemma 2.10, and claim (2) follows from Lemma 2.18.

Theorem 2.20. $\operatorname{St}^{\text {conj }}(L)=\operatorname{Conj}(G) \rtimes S t(L)$. 
Proof. First we show that $\operatorname{Conj}(G) \subseteq \operatorname{St}^{\operatorname{conj}}(L)$. As $\operatorname{Conj}(G)$ is generated by automorphisms of the form given in Definition 1.3 it suffices to show that $\phi=\alpha_{C}(y)$ belongs to $\mathrm{St}^{\mathrm{conj}}(L)$, where $y \in X$ and $C$ is a connected component of the full subgraph on $X \backslash y^{\perp}$. Let $V \in L$, so $V=T^{\perp}$ for some $T \in L$. If $y \in V$ then $G(Y)^{\phi}=G(V)$, so assume $y \notin V$. Let $v_{1}, v_{2} \in V$. If $v_{1} \in y^{\perp}$ then $v_{1}^{\phi}=v_{2}^{\phi}$, so assume $v_{i} \notin y^{\perp}$ for $i=1,2$. Now $y \notin V$ implies there exists some $t \in T$ such that $[y, t] \neq 1$. As $v_{i} \in T^{\perp}$ and $v_{i} \notin y^{\perp}$ it follows that $v_{1}, v_{2}$ and $t$ lie in a connected component of $\Gamma\left(X \backslash y^{\perp}\right)$. In particular $v_{1}^{\phi}=v_{2}^{\phi}$. Therefore either $G(V)^{\phi}=G(V)$ or $G(V)^{\phi}=G(V)^{y}$ and so $\phi \in \mathrm{St}^{\mathrm{conj}}(L)$, as required.

Next we show that $\operatorname{Conj}(G) \triangleleft \mathrm{St}^{\text {conj }}(L)$. It suffices to show that $\theta^{-1} \phi \theta \in$ $\operatorname{Conj}(G)$, where $\phi$ is defined as above and $\theta \in \operatorname{St}^{\text {conj }}(L)$. Let $x \in X$ and let $g \in G$ such that $G(\operatorname{cl}(x))^{\theta}=G(\operatorname{cl}(x))^{g}$. Then $G(\operatorname{cl}(x))^{\theta^{-1}}=G(\operatorname{cl}(x))^{h}$, where $h=\left(g^{-1}\right)^{\theta^{-1}}$. Thus $x^{\theta^{-1}}=w^{h}$ for some $w \in G(\operatorname{cl}(x))$, so $x=\left(w^{\theta}\right)^{h \theta}$ and $x^{g}=w^{\theta}$. Now $x^{\theta^{-1} \phi \theta}=\left(w^{h}\right)^{\phi \theta}=\left(\left(w^{\phi}\right)^{h^{\phi}}\right)^{\theta}$, so

$$
x^{\theta^{-1} \phi \theta}= \begin{cases}\left(\left(w^{y}\right)^{h^{\phi}}\right)^{\theta}=\left(w^{\theta}\right)^{y^{\theta} h^{\phi \theta}}=x^{g y^{\theta} h^{\phi \theta}} & \text { if } \operatorname{cl}(x) \subseteq C \cup y^{\perp}, \\ \left(w^{h^{\phi}}\right)^{\theta}=\left(w^{\theta}\right)^{h^{\phi \theta}}=x^{g h^{\phi \theta}} & \text { otherwise. }\end{cases}
$$

Therefore $\theta^{-1} \phi \theta$ is a conjugating automorphism.

Now we shall show that any element $\theta \in \mathrm{St}^{\mathrm{conj}}(L)$ can be expressed as $\theta=\tau \phi$ for some $\tau \in \operatorname{Conj}(G)$ and $\phi \in \operatorname{St}(L)$. Fix $\theta \in \operatorname{St}^{\text {conj }}(L)$ and for all $Y \in L$ fix $g_{Y} \in G$ such that $G(Y)^{\theta}=G(Y)^{g_{Y}}$. Without loss of generality we may choose $g_{Y}$ so that none of its left divisors belong to $G(Y)$ or to $C_{G}(Y)=G\left(Y^{\perp}\right)$. Given two non-empty closed sets $Y, Z \in L$ with $Y \subseteq Z$, we claim that $g_{Y} g_{Z}^{-1}=a b$ where $a \in G(Z)$ and $b \in C_{G}(Y)$. To see this suppose that $u \in G(Y)$ and let $r \in G(Y)$, $s \in G(Z)$ such that $u^{\theta}=r^{g_{Y}}=s^{g_{Z}}$. From [6], Corollary 2.4, and the choice of $g_{Y}$ and $g_{Z}$ there exist $c, c^{\prime}, d_{1}, d_{1}^{\prime}, d_{2}, d_{2}^{\prime}, v \in G$ such that $g_{Y}=c \circ d_{2}, g_{Z}=c^{\prime} \circ d_{2}^{\prime}$, $r=d_{1}^{-1} \circ v \circ d_{1}, s=d_{1}^{\prime-1} \circ v \circ d_{1}^{\prime}$, and with $d=d_{1} \circ d_{2}$ and $d^{\prime}=d_{1}^{\prime} \circ d_{2}^{\prime}$, $r^{g_{Y}}=d^{-1} \circ v \circ d$ and $s^{g_{Z}}=d^{\prime^{-1}} \circ v \circ d^{\prime}$.

By definition of $\theta$ for $x \in Y$ there exists $u \in G(Y)$ such that $u^{\theta}=x^{g_{Y}}$. We may then take $r=x$ and $s \in G(Z)$ such that $u^{\theta}=s^{g_{Z}}=x^{g_{Y}}$. In this case we have $r=x=v$ and so $d_{1}=1$ and, again by [6], Corollary 2.4, $c, c^{\prime} \in C_{G}(x)$. Allowing $x$ to range over $Y$ we see that $c, c^{\prime} \in C_{G}(Y)$, and by choice of $g_{Y}$ it follows that $c=1$ and $g_{Y}=d_{2}$. Now, with $r=x \in Y$, again we have $d_{2}^{-1} \circ x \circ d_{2}=r^{g_{Y}}=$ $s^{g Z}=d^{\prime-1} \circ x \circ d^{\prime}$, so $d^{\prime}=d_{2}$. As $\alpha\left(d_{1}^{\prime}\right) \subseteq \alpha(s) \subseteq Z$ we have $d_{1}^{\prime} \in G(Z)$. If $d_{1}^{\prime}$ has a left divisor in $G(Y)$ then so does $g_{Y}$, and $c^{\prime} \notin C_{G}(Z)$ since it is a left divisor of $g_{Z}$. This completes the proof of the claim as $g_{Y} g_{Z}^{-1}=d_{1}^{\prime} c^{-1}$.

Next we use $\theta$ to construct a homomorphism from $G$ to itself and subsequently show that this homomorphism is an element of $\operatorname{St}(L)$. Let $x \in X$, so $G(\operatorname{cl}(x))^{\theta}=$ $G(\operatorname{cl}(x))^{g_{x}}$. Then there exists $u_{x} \in G(\operatorname{cl}(x))$ such that $x^{\theta}=u_{x}^{g_{x}}$. Define a map $\phi: X \rightarrow G$ by $x^{\phi}=u_{x}$ for all $x \in X$. Suppose that $x, y \in X$ with $[x, y]=1$. Then $x, y \in x^{\perp} \cap y^{\perp}$, hence $\operatorname{cl}(x) \cup \operatorname{cl}(y) \subseteq x^{\perp} \cap y^{\perp}$. Let $Z=x^{\perp} \cap y^{\perp}$ and 
so $G(Z)^{\theta}=G(Z)^{g_{Z}}$. As $\operatorname{cl}(x) \subseteq Z$ we have, from the above, $g_{x}=a b g_{Z}$, with $a \in G(Z)$ and $b \in C_{G}(\operatorname{cl}(x))=G\left(x^{\perp}\right)$. Thus $a b \in G\left(x^{\perp}\right)$ and $u_{x}^{a b}=u_{x}$. Hence $u_{x}^{g_{x}}=u_{x}^{g_{Z}}$ and similarly $u_{y}^{g_{y}}=u_{y}^{g_{Z}}$. Because $\theta$ is an automorphism, we have $1=\left[u_{x}^{g_{x}}, u_{y}^{g_{y}}\right]=\left[u_{x}, u_{y}\right]^{g z}$, so $\left[u_{x}, u_{y}\right]=1$. Therefore $\phi$ extends to an endomorphism of $G$.

The next step is to show that $\phi$ is surjective. To this end suppose that $y, z \in X$ and $\operatorname{cl}(y) \subseteq \operatorname{cl}(z)$. If $u \in \operatorname{cl}(y)$ then $[u, v]=1$ for all $v \in \operatorname{cl}(z)$ since $\operatorname{cl}(z) \subseteq z^{\perp} \subseteq y^{\perp}$. We have $g_{y}=a b g_{z}$, where $a \in G(\operatorname{cl}(z))$ and $b \in G\left(y^{\perp}\right)$. Hence $u_{y}^{a b}=u_{y}$ and so $u_{y}^{g_{y}}=u_{y}^{g_{z}}$. Now let $x \in X$. As $G(\operatorname{cl}(x))^{\theta}=G(\operatorname{cl}(x))^{g_{x}}$ there exists $w \in G(\operatorname{cl}(x))$ such that $w^{\theta}=x^{g_{x}}$. Assume that $w=y_{1}^{\varepsilon_{1}} \ldots y_{n}^{\varepsilon_{n}}$ for some $y_{i} \in \operatorname{cl}(x)$ and $\varepsilon_{i}= \pm 1$. Let $u_{i}=u_{y_{i}}$. Then $\operatorname{cl}\left(y_{i}\right) \subseteq \operatorname{cl}(x)$, hence $y_{i}^{\theta}=u_{i}^{g_{x}}$ from the preceding argument, and $w^{\theta}=\left(u_{1}^{\varepsilon_{1}} \ldots u_{n}^{\varepsilon_{n}}\right)^{g_{x}}=x^{g_{x}}$. Hence $w^{\phi}=u_{1}^{\varepsilon_{1}} \ldots u_{n}^{\varepsilon_{n}}=x$ and $\phi$ is surjective.

To show that $\phi$ is injective consider the automorphism $\theta^{-1}$ and let $h_{x}=\left(g_{x}^{-1}\right)^{\theta^{-1}}$ for all $x \in X$. Choose, for all $x \in X$, an element $k_{x} \in G$ and $v_{x} \in G(\operatorname{cl}(x))$ such that $G(\operatorname{cl}(x))^{\theta^{-1}}=G(\operatorname{cl}(x))^{k_{x}}, x^{\theta^{-1}}=v_{x}^{k_{x}}$ and $k_{x}$ has no left divisor in $G(\operatorname{cl}(x))$. Then, as in the case of $\theta$ and $\phi$ above, the map $\bar{\phi}: x \rightarrow v_{x}$ extends to an endomorphism of $G$. Moreover $h_{x}=j_{x} k_{x}$ for some $j_{x} \in G\left(x^{\perp}\right)$. Suppose that $u_{x}=y_{1}^{\varepsilon_{1}} \ldots y_{n}^{\varepsilon_{n}}$, where $y_{i} \in \operatorname{cl}(x)$. Write $v_{i}=v_{y_{i}}=y_{i}^{\bar{\phi}}$ for $i=1, \ldots, n$. Then, from the above, $y_{i}^{\theta^{-1}}=v_{i}^{k_{x}}=v_{i}^{h_{x}}$ since $\operatorname{cl}\left(y_{i}\right) \subseteq \operatorname{cl}(x), v_{i} \in \operatorname{cl}\left(y_{i}\right)$ and $j_{x} \in G\left(x^{\perp}\right)$. Now $x=x^{\theta \theta^{-1}}=\left(u_{x}^{g_{x}}\right)^{\theta^{-1}}=\left(u_{x}^{\theta^{-1}}\right)^{h_{x}^{-1}}=\left(v_{1}^{\varepsilon_{1} h_{x}} \ldots v_{n}^{\varepsilon_{n} h_{x}}\right)^{h_{x}^{-1}}=v_{1}^{\varepsilon_{1}} \ldots v_{n}^{\varepsilon_{n}}$, so $x^{\phi \bar{\phi}}=u_{x}^{\bar{\phi}}=v_{1}^{\varepsilon_{1}} \ldots v_{n}^{\varepsilon_{n}}=x$. It follows that $\phi$ is a bijection and hence is an automorphism. By definition $\phi$ maps $G(\operatorname{cl}(x))$ to itself for all $x \in X$, and so belongs to $\operatorname{St}(L)$.

Now define $l_{x}=g_{x}^{\phi^{-1}}$ for all $x \in X$. Then $x^{\theta}=u_{x}^{g_{x}}=\left(x^{\phi}\right)^{l_{x}^{\phi}}=\left(x^{l_{x}}\right)^{\phi}$, so $\tau=\theta \phi^{-1}$ is a conjugating automorphism. Note that if $\rho \in \operatorname{Conj}(G) \cap \operatorname{St}(L)$ then $x^{\rho}=x^{w_{x}}$ for some $w_{x} \in G$, and $G(\operatorname{cl}(x))^{\rho}=G(\operatorname{cl}(x))$. Therefore, $x^{w_{x}} \in$ $G(\operatorname{cl}(x))$. It follows that $w_{x} \in G\left(x^{\perp}\right)$, so $x^{\rho}=x$ and $\rho$ is the identity map. Hence $\operatorname{Conj}(G) \cap \operatorname{St}(L)=\{1\}$. Now suppose that $\tau, \tau^{\prime} \in \operatorname{Conj}(G)$ and $\phi, \phi^{\prime} \in \operatorname{St}(L)$. Then $\tau \phi=\tau^{\prime} \phi^{\prime}$ implies $\tau^{\prime-1} \tau=\phi^{\prime} \phi \in \operatorname{Conj}(G) \cap \operatorname{St}(L)$, so $\tau=\tau^{\prime}$ and $\phi=\phi^{\prime}$. What we have shown is that every element $\theta \in \mathrm{St}^{\mathrm{conj}}(L)$ can be uniquely expressed as $\theta=\tau \phi$ with $\tau \in \operatorname{Conj}(G)$ and $\phi \in \operatorname{St}(L)$. The theorem now follows.

\section{References}

[1] A. Baudisch, Kommutationsgleichungen in semifreien Gruppen. Acta Math. Acad. Sci. Hungar. 29 (1977), 235-249. Zbl 0381.20027 MR 0463300

[2] R. Charney, J. Crisp, and K. Vogtmann, Automorphisms of 2-dimensional right-angled Artin groups. Geom. Topol. 11 (2007), 2227-2264. Zbl 1152.20032 MR 2372847

[3] R. Charney and K. Vogtmann, Automorphisms of higher-dimensional right-angled Artin groups. Preprint 2007. arXiv:0709.2700v2 [math.GR] 
[4] R. Charney and K. Vogtmann, Automorphism groups of right-angled Artin groups. In Guido's book of conjectures, Monogr. Enseign. Math. 40, L'Enseignement Mathématique, Geneva, 2008; L'Enseign. Math. 54 (2008), 71-72.

[5] G. Duchamp and D. Krob, Partially commutative Magnus transformations. Internat. J. Algebra Comput. 3 (1993), 15-41. Zbl 0820.20037 MR 1214003

[6] A. J. Duncan, I. V. Kazachkov, and V. N. Remeslennikov, Centraliser dimension of partially commutative groups. Geom. Dedicata 120 (2006), 73-97. Zbl 1172.20303 MR 2252895

[7] A. J. Duncan, I. V. Kazachkov and V. N. Remeslennikov, Orthogonal systems in finite graphs. Sib. Èlektron. Mat. Izv. 5 (2008), 151-176. MR 2586627

[8] A. J. Duncan, I. V. Kazachkov, and V. N. Remeslennikov, Parabolic and quasiparabolic subgroups of free partially commutative groups. J. Algebra 318 (2007), 918-932. Zbl 1183.20026 MR 2371978

[9] E. S. Esyp, I. V. Kazatchkov, and V. N. Remeslennikov, Divisibility theory and complexity of algorithms for free partially commutative groups. In Groups, languages, algorithms, Contemp. Math. 378, Amer. Math. Soc., Providence, RI, 2005, 319-348. Zbl 1160.20306 MR 2159318

[10] M. Gutierrez, A. Piggott, and K. Ruane, On the automorphisms of a graph product of abelian groups. Groups Geom. Dyn., to appear. arXiv:0710.2573

[11] M. R. Laurence, A generating set for the automorphism group of a graph group. J. London Math. Soc. (2) 52 (1995), 318-334. Zbl 0836.20036 MR 1356145

[12] H. Servatius, Automorphisms of graph groups. J. Algebra 126 (1989), 34-60. Zbl 0682.20022 MR 1023285

Received March 14, 2008; revised September 24, 2009

A. J. Duncan, School of Mathematics and Statistics, Newcastle University, Newcastle upon Tyne, NE1 7RU, United Kingdom

E-mail: a.duncan@ncl.ac.uk

I. V. Kazachkov, Department of Mathematics and Statistics, McGill University, Burnside 1029, 805 Sherbrooke Street West, Montreal, QC, H3A 2K6, Canada

E-mail: ilya.kazachkov@gmail.com

V. N. Remeslennikov, Department of Mathematics, Omsk State University, 55-A Prospect Mira, Omsk , 644077 Omsk, Russia

E-mail: remesl@ofim.oscsbras.ru 